\title{
DEVELOPMENT OF AN EXTENDED EXTERIOR DIFFERENTIAL CALCULUS
}

\author{
BY \\ HARLEY FLANDERS
}

\section{INTRODUCTION}

The purpose of this paper is to set up an algebraic machinery for the theory of affine connections on differentiable manifolds and to demonstrate by means of several applications the scope and convenience of this mechanism.

We shall associate with a manifold a series of spaces, best described as spaces of multivectors with exterior differential form coefficients, and shall exhibit the algebraic relations between these spaces. It is possible to consider, in a more general fashion, spaces of tensors with differential form coefficients; this is done, in fact, in Cartan [3, Chap. VIII, Sec. II] $\left({ }^{1}\right)$, where their use is justified by means of geometrical considerations.

We shall define an affine connection as a certain kind of operator on the space of ordinary vector fields to the space of vector fields with differential one-form coefficients. It will be seen that this is simply another formulation of the classical definition. One of our basic results (Theorem 7.1) is that an affine connection induces an operator on each of the series of spaces just mentioned.

In Chapter I, we shall summarize the facts that we need about differentiable manifolds and introduce some notation. Chapter II is devoted to the algebraic structure of the series of spaces $\mathcal{T}_{q}^{p}$ that we introduce. In Chapter III, we give the calculus associated with an affine connection and applications to a number of identities. In the final chapter, we discuss some applications of our calculus to Riemannian geometry, in particular to the "curvatura integra" of S. Chern.

We hope in the future to give applications of this theory to other parts of differential geometry, possibly to the theory of harmonic integrals and to the theory of Lie groups.

\section{Chapter I. Differentiable manifolds}

1. Basic definitions. Let $\mathfrak{M}$ denote an $n$-dimensional differentiable manifold. Usually we shall assume that $\mathfrak{M}$ is of class $C^{\infty}$, i.e., $\mathfrak{M}$ bears an infinitely differentiable structure; however, we shall have occasion for a few remarks on the $C^{\omega}$ case, i.e., when $\mathfrak{M}$ bears an analytic structure (Chevalley [9]). The definitions and results we shall now state for $C^{\infty}$ structures carry over

Presented to the Society, December 29, 1952; received by the editors August 25, 1952.

(1) Numbers in brackets refer to the bibliography at the end of this paper. 
with slight modifications to the analytic case. The reader may consult Chevalley, loc. cit., for details.

The $C^{\infty}$ structure of $\mathfrak{M}$ carries along with it a space $C(\mathfrak{M})$ of all infinitely differentiable real-valued functions on $\mathfrak{M}$. Let $P$ be a point of $\mathfrak{M}$. A tangent vector at $P$ is a real-valued function $v$ on $C(M)$ satisfying

$$
\begin{aligned}
\boldsymbol{v}(f+g) & =\boldsymbol{v}(f)+\boldsymbol{v}(g), \\
\boldsymbol{v}(a f) & =a \boldsymbol{v}(f), \\
\boldsymbol{v}(f g) & =\boldsymbol{v}(f) g(P)+f(P) \boldsymbol{v}(g) .
\end{aligned}
$$

Here $f, g \in C(\mathfrak{M})$ and $a$ is real. The set of all tangent vectors at $P$ forms a linear space which we shall denote by $\mathfrak{I}_{P}$. It is known that $\mathfrak{I}_{P}$ is an $n$-dimensional space and that a basis of $\mathfrak{T}_{P}$ may be obtained as follows $\left({ }^{2}\right)$. Let $\mathfrak{U}$ be a local coordinate neighborhood on $\mathfrak{M}$, containing $P$, and with coordinate functions $x^{1}, \cdots, x^{n}$. Define vectors $e_{i}$ at $P$ by $e_{i}(f)=\left(\partial f / \partial x^{i}\right)_{P}$. Then $e_{1}, \cdots, e_{n}$ is a basis of $\mathfrak{I}_{P}$. The dual space $\mathfrak{F}_{P}$ of $\mathfrak{T}_{P}$ is usually called the space of onejorms at $P$. The basis of $\mathfrak{F}_{P}$ which is dual to the basis $\boldsymbol{e}_{1}, \cdots, \boldsymbol{e}_{n}$ is denoted $d x^{1}, \cdots, d x^{n}$. Thus if $\left[v, w^{\prime}\right]$ denotes the application of a one-form $w^{\prime}$ to a vector $v$, then $\left[e_{i}, d x^{j}\right]=\delta_{i}^{j}$, the Kronecker $\delta$.

If $v$ is any tangent vector at $P$, then $v=\sum a^{i} e_{i}$, with unique constants $a^{i}$. A mapping $X$ which sends each point $P$ of $\mathfrak{M}$ into a tangent vector $X(P)$ in $\mathfrak{T}_{P}$ is called a vector field (or infinitesmal transformation) provided that in each local coordinate neighborhood $\mathfrak{u}$ the expression $X(P)=\sum a^{i}\left(x^{1}, \cdots, x^{n}\right) \boldsymbol{e}_{i}$ defines $C^{\infty}$ functions $a^{i}(x)$ on a region of euclidean space $E_{n}$. When there is no danger of confusion, we shall refer to such a vector field simply as a vector on $\mathfrak{M}$ and denote it by the same symbol $v$ as we used for a vector at a point. In the same manner we define a form field or differential form of degree one, $\omega=\sum a_{i} d x^{i}$.

The following notation will be useful. Let $\mathcal{F}_{0}=\mathcal{F}_{0}(\mathfrak{M})$ denote the ring of all $C^{\infty}$ functions on $\mathfrak{M}$. We have previously called this $C(\mathfrak{M})$, but now wish to include it in the hierarchy of $q$-forms. Let $\mathcal{T}=\mathcal{T}(\mathfrak{M})$ be the space of all vectors (i.e., vector fields) on $\mathfrak{M}$. This space may be considered as a linear space over the ring of operators $\mathcal{F}_{0}$ (see Bourbaki [1]).

In much of this paper we shall work locally. Let $\mathfrak{U}$ be a local coordinate neighborhood of $\mathfrak{M}$. Then $\mathfrak{U}$ is an open connected subset of $\mathfrak{M}$ so that $\mathfrak{U}$ itself is an $n$-dimensional manifold. Thus all that has been said may be applied to $\mathfrak{U}$. However the fact that $\mathfrak{U}$ may be coordinatized implies the special property that $\mathfrak{T}$ is an $n$-dimensional space over the ring $\mathcal{F}_{0}$.

2. Two important bundles. Consider the space $\mathfrak{B}_{1}=U_{P} \mathfrak{T}_{P}$. This is a fiber bundle (Steenrod [12]), called the tangent bundle of the manifold $\mathfrak{M}$, and it again carries a $C^{\infty}$ structure; in fact, from the expression $v=\sum a^{i} e_{i}$ we ex-

(2) This result is given in Chevalley, loc. cit., for $C^{\omega}$ structures. Since it is not readily accessible as we have stated it, we shall include a short proof in the appendix to this chapter. 
tract the local coordinate system $x^{1}, \cdots, x^{n}, a^{1}, \cdots, a^{n}$ on the neighborhood of $\mathfrak{B}_{1}$ consisting of $\bigcup_{P} \in \mathfrak{u} \mathfrak{I}_{P}$. A vector field on $\mathfrak{M}$ is the same thing as a $C^{\infty}$ cross section of $\mathfrak{B}_{1}$.

Next, by a frame at $P$ we mean any basis $\mathbf{e}_{1}, \cdots, \mathbf{e}_{n}$ of the linear space $\mathfrak{I}_{P}$. The set of all frames at all points $P$ of $\mathfrak{M}$ forms a new bundle $\mathfrak{B}_{n}$ called the frame bundle of $\mathfrak{M}$. Since one passes from one frame to another in $\mathfrak{I}_{P}$ by the most general nonsingular linear transformation on an $n$-dimensional linear space, it follows that $\mathfrak{B}_{n}$ has dimension $n+n^{2}$. A cross section of $\mathfrak{B}_{n}$ is called a moving frame on $\mathfrak{M}$. The application of such frames to Riemannian geometry has been given in Flanders [11].

3. Multivectors and forms. Over the vector space $\mathfrak{T}_{P}$ associated with a point $P$ of $\mathfrak{M}$, one may form the space $\Lambda^{p} \mathfrak{T}_{P}$ of $p$-vectors at $P$. (See Cartan $[3,4]$ and Bourbaki $[2]$.) In the same way one may form the space $\Lambda^{a} \mathfrak{F}_{P}$ of $q$-forms at $P$. Each of these gives rise to a corresponding bundle (on which, again, the full linear group acts), whose $C^{\infty}$ cross sections are called $p$-vector fields and differential form fields of degree $q$ respectively.

It is important to note that the ring $C(\mathfrak{M})$ acts both on the linear space $\mathcal{T}^{p}(\mathfrak{M})$ of all $p$-vector fields and on the linear space $\mathcal{F}_{q}(\mathfrak{M})$ of all $q$-forms ( $q$-form fields) on $\mathfrak{M}$. This remark helps to clarify the following assertion. Let us restrict attention to a local coordinate neighborhood $\mathfrak{U}$ on $\mathfrak{M}, \mathcal{F}_{0}=C(\mathfrak{U})$ as above, $\mathcal{T}^{p}=\mathcal{T}^{p}(\mathfrak{U}), \mathcal{F}_{q}=\mathcal{F}_{q}(\mathfrak{U})$. The space $\mathcal{T}^{1}=\mathcal{T}$ is then an $n$-dimensional space over $\mathcal{F}_{0}$ and it is true that $\mathcal{T}^{p}=\Lambda^{p} \mathcal{T}$ formed over this coefficient domain $\mathcal{F}_{0}$. In the same way $\mathcal{F}_{q}=\wedge^{q} \mathcal{F}_{1}$.

4. Appendix. In this section we shall sketch a proof of the assertions in $\S 1$ about the structure of the space $\mathfrak{I}_{P}$. The crux of the proof is contained in the following lemma, whose proof was communicated to the author by Professor H. F. Bohnenblust.

Lemma 4.1. Let $f(x)$ be a $C^{\infty}$ function defined in a neighborhood of 0 on the real axis. Assume $f(0)=0$. Then there is a $C^{\infty}$ function $g(x)$ defined in the same neighborhood such that $g(x)=f(x) / x$ for $x \neq 0$ and $g(0)=f^{\prime}(0)$.

It should be noted that this is trivial for $f(x)$ analytic, and it is this fact which is used in Chevalley [9, p. 78] to prove the corresponding facts about $\mathfrak{I}_{P}$ in the case of analytic manifolds. To prove our lemma, we simply write down the answer. Set

$$
g(x)=\int_{0}^{1} f^{\prime}(t x) d t
$$

This function is easily seen to have the required properties.

Corollary. Let $F\left(x^{1}, \cdots, x^{n}\right)$ be a $C^{\infty}$ function in a star-shaped neighborhood $\mathfrak{U}$ of $a$ point $a=\left(a^{1}, \cdots, a^{n}\right)$ of $E_{n}$. Then there exist $C^{\infty}$ functions $G_{i}(x)\left(i=1, \cdots, n, x=\left(x^{1}, \cdots, x^{n}\right)\right)$ on the same neighborhood such that 
$F(\mathbf{x})=\sum G_{i}(\mathbf{x})\left(x^{i}-a^{i}\right)$ and $G_{i}(\mathbf{a})=\left(\partial F / \partial x^{i}\right)_{\mathbf{a}}$.

This is proved by applying the lemma to the function $f(u)$ $=F(\mathbf{a}+u(\mathbf{x}-\mathbf{a}))$.

The proof of our assertions about $\mathfrak{I}_{P}$ is now almost identical with that in Chevalley, loc. cit., so we shall omit further details.

\section{Chapter II. Vectors With Form COEFficients}

5. Algebraic properties. In this section we shall consider the tensor products

$$
\mathcal{T}_{q}^{p}=\mathcal{F}_{q} \otimes \mathcal{T}^{p}
$$

These spaces may be considered from two points of view: either as tensor products of the spaces $\mathcal{F}_{q}$ and $\mathcal{T}^{p}$ over the ring $\mathcal{F}_{0}$, or as cross sections of the bundle of all elements of all $\left(\Lambda^{q} \mathfrak{F}_{P}\right) \otimes\left(\Lambda^{p} \mathfrak{T}_{P}\right)$; there the latter tensor product is taken over the field of reals. At any rate, the ring $\mathcal{F}_{0}$ acts as a coefficient ring for the spaces $\mathcal{T}_{q}^{p}$.

Let us set $\mathcal{F}=\sum \oplus \mathcal{F}_{q}, \mathcal{T}=\sum \oplus \mathcal{T}^{p}$. Each of these spaces is an algebra over $\mathcal{F}_{0}$, where multiplication is the Grassmann product. This implies, passing to homogeneous components, the existence of an operation on $\mathcal{T}_{q}^{p} \times \mathcal{T}_{q^{\prime}}^{p^{\prime}}$ to $\mathcal{T}_{q+q^{\prime}}^{p+p^{\prime}}$ given by linearity and

$$
(\omega \boldsymbol{v})(\eta \boldsymbol{w})=(\omega \eta) v w, \quad \text { where } \omega \in \mathcal{F}_{\boldsymbol{q}}, \eta \in \mathcal{F}_{\boldsymbol{q}^{\prime}}, \boldsymbol{v} \in \mathcal{T}^{p}, \boldsymbol{w} \in \mathcal{T}^{p^{\prime}} .
$$

This operation is associative and distributive, and obeys the following commutation rule

$$
v w=(-1)^{p p^{\prime}+q q^{\prime}}{ }_{w v} \text { for } \quad v \in \mathcal{T}_{q}^{p} \text { and } w \in \mathcal{T}_{q^{\prime}}^{p^{\prime}}
$$

6. The displacement vector. Let $e_{1}, \cdots, e_{n}$ be a moving frame on a local coordinate neighborhood $\mathfrak{U}$ of $\mathfrak{M}$. Let $\sigma^{1}, \cdots, \sigma^{n}$ be a dual basis of one-forms. We set

$$
d P=\sigma^{1} e_{1}+\cdots+\sigma^{n} e_{n}
$$

so that $d P \in \mathcal{T}_{1}^{1}$. It is clear that $d P$ is intrinsic, i.e., it is independent of the particular moving frame which is used to define it, and we shall call it the displacement vector of $\mathfrak{M}$. The geometric meaning of $d P$ is easily seen as follows (Cartan [3]). Let $P=P(t)$ be a moving particle on $\mathfrak{M}$, where the real variable $t$ denotes time. Then along the trajectory $c$ of $P$ the $\sigma^{i}$ contract to one-forms in $t$ and the velocity vector of $P$ is given by

$$
\frac{d P}{d t}=\frac{\sigma^{1}}{d t} e_{1}+\cdots+\frac{\sigma^{n}}{d t} e_{n} .
$$

As examples of our multiplication we have 


$$
d P^{2}=2 \sum_{i<j} \sigma^{i} \sigma^{j} \boldsymbol{e}_{i} \boldsymbol{e}_{j} \text { and } d P^{n}=(n !) \sigma^{1} \cdots \sigma^{n} \boldsymbol{e}_{1} \cdots \mathbf{e}_{n} .
$$

\section{Chapter III. Affine connection}

7. Definition. The exterior differentiation operator $d$ maps $\mathcal{F}_{q}$ into $\mathcal{F}_{q+1}$ and is characterized by the following properties: (1) linearity, (2) $d d=0$, (3) $d f=\sum\left(\partial f / \partial u^{i}\right) d u^{i}$ when $f$ is a function on $\mathfrak{u}$, a neighborhood coordinatized by $u^{1}, \cdots, u^{n}$, and (4) $d(\omega \eta)=d \omega \eta+(-1)^{q} \omega d \eta$, where $\omega \in \mathcal{F}_{q}$.

Definition 7.1. An affine connection on $\mathfrak{M}$ is an operator $d$ on $\mathcal{T}_{0}^{1}$ into $\mathcal{T}_{1}^{1}$ satisfying the two properties

$$
\begin{aligned}
d(v+w) & =d v+d w \\
d(f v) & =d f v+f d v
\end{aligned}
$$

That this definition is equivalent to the classical one as given in Cartan [5] or Eisenhart [10] is a fact whose demonstration we shall defer to $\$ 12$. The main result of this section is the demonstration that given an affine connection $\boldsymbol{d}$, then the operator $\boldsymbol{d}$ induces unique operators $\boldsymbol{d}$ on each $\tau_{q}^{p}$ to $\mathcal{T}_{q+1}^{p}$ satisfying, in addition to the obvious linearity, a condition consistent with the condition (b) of the definition.

THEOREM 7.1. If $\boldsymbol{d}$ is an affine connection on $\mathfrak{M}$, then there exists a unique collection of operators, also denoted $d$, one on each space $\mathcal{T}_{q}^{p}$ to $\mathcal{T}_{q+1}^{p}$, and satisfying the following three properties:

(a) $d(v+w)=d v+d w$ for $v, w \in \mathcal{T}_{q}^{p}$,

(b) $d(v w)=d v w+(-1)^{q} v d w$ for $v \in \mathcal{T}_{q}^{p}, w \in \mathcal{T}_{s}^{r}$,

(c) $\boldsymbol{d}$ coincides with the given affine connection on $\mathcal{T}_{0}^{1}$, and $\boldsymbol{d}$ coincides with exterior differentiation on $\mathcal{G}_{q}^{0}=\mathcal{F}_{q}$.

Proof. We begin with the uniqueness proof. If $v \in \mathcal{T}_{q}^{p}$, then $v$ is a linear combination of vectors of the form $w=\omega \mathbf{x}_{1} \cdots \mathbf{x}_{p}$, where $\omega \in \mathcal{T}_{q}^{0}$ and $\mathbf{x}_{i} \in \mathcal{T}_{0}^{p}$. If an operator $d$ satisfying the conditions of the theorem exists, then we have $d w=d \omega x_{1} \cdots x_{p}+(-1)^{q} \omega \sum x_{1} \cdots x_{i-1} d x_{i} x_{i+1} \cdots x_{p}$. This, combined with linearity, yields the uniqueness of $d$.

To prove the existence of $\boldsymbol{d}$, we shall begin by defining $\boldsymbol{d}$ on the spaces $\tau_{0}^{p}$ of $p$-vectors with function coefficients. Consider the mapping

$$
\left(x_{1}, \cdots, x_{p}\right) \rightarrow \sum_{1}^{p} x_{1} \cdots x_{i-1} d x_{i} x_{i+1} \cdots x_{p}
$$

on $p$-tples of vectors of $\mathcal{T}_{0}^{1}$ into $\mathcal{T}_{1}^{p}$. This mapping is alternating and multilinear, hence it follows from Bourbaki [2, Scholium, p. 64] that there is a linear mapping $\boldsymbol{d}$ on $\mathcal{T}_{0}^{p}$ to $\mathcal{T}_{1}^{p}$ such that

$$
d\left(x_{1} \cdots x_{p}\right)=\sum_{1}^{p} x_{1} \cdots x_{i-1} d x_{i} x_{i+1} \cdots x_{p}
$$


for any vectors $x_{1}, \cdots, x_{p}$ in $\mathcal{T}_{0}^{1}$. To show that (b) is satisfied for $v \in \mathcal{F}_{0}^{p}$, $\boldsymbol{w} \in \mathcal{T}_{0}^{r}$, we observe that by linearity it suffices to consider the case $v=x_{1} \ldots$ $x_{p}, w=y_{1} \cdots y_{r}$ where the $x_{i}$ and $y_{j}$ are in $\mathcal{T}_{0}^{1}$. But property (b) is immediate in view of the last formula.

We complete the proof by defining $\boldsymbol{d}$ on $\mathfrak{T}_{q}^{p}$. To do this, we consider the mapping

$$
(\omega, v) \rightarrow d \omega v+(-1)^{q} \omega d v
$$

which is on $\mathcal{T}_{a}^{0} \times \mathcal{T}_{0}^{p}$ into $\mathcal{T}_{a+1}^{p}$. This mapping is bilinear and so we may apply the result in Bourbaki [2, Scholium, p. 7] to prove that there is a linear mapping $d$ on $\mathcal{T}_{q}^{p}=\mathcal{T}_{q}^{0} \otimes \mathcal{T}_{0}^{p}$ into $\mathcal{T}_{q+1}^{p}$ such that

$$
d(\omega v)=d \omega v+(-1)^{q} \omega d v
$$

for any $\omega \in \mathcal{T}_{q}^{0}$ and any $v \in \mathcal{T}_{0}^{p}$.

It now remains to verify property (b). By linearity, it suffices to do this in the following special case: $v=\omega u, w=\eta z$, where $\omega \in \mathcal{T}_{q}^{0}, \eta \in \mathcal{T}_{s}^{0}, u \in \mathcal{T}_{0}^{p}$, $z \in \mathcal{T}_{0}^{r}$. In this case we have

$$
\begin{aligned}
d(v w) & =d[(\omega u)(\eta z)]=d[(\omega \eta)(u z)] \\
& =d(\omega \eta)(u z)+(-1)^{q+o}(\omega \eta) d(u z) \\
& =\left(d \omega \eta+(-1)^{q} \omega d \eta\right)(u z)+(-1)^{q+s}(\omega \eta)(d u z+u d z) \\
& =(d \omega u)(\eta z)+(-1)^{q}(\omega u)(d \eta z)+(-1)^{q}(\omega d u)(\eta z)+(-1)^{q+\bullet}(\omega u)(\eta d z) \\
& =\left(d \omega u+(-1)^{q} \omega d u\right)(\eta z)+(-1)^{q}(\omega u)\left(d \eta z+(-1)^{\imath} \eta d z\right) \\
& =d v w+(-1)^{q} v d w .
\end{aligned}
$$

In this computation we have used the relation $\eta d u=(-1)^{\bullet} d u \eta$. This is a consequence of the commutation rule (5.3).

We may now simplify our notation by using the same symbol $d$ to denote exterior differentiation $d$, the (given) affine connection $d$, and all of the derived operators $\boldsymbol{d}$ of the last theorem.

8. Curvature and torsion. The following matrix notation will greatly simplify our work. If $e_{1}, \cdots, e_{n}$ is a moving frame with the dual basis of forms $\sigma^{1}, \cdots, \sigma^{n}$, we shall set

$$
e=\left(\begin{array}{c}
e_{1} \\
\vdots \\
e_{n}
\end{array}\right), \quad \sigma=\left(\sigma^{1} \cdots \sigma^{n}\right)
$$

and we make the convention of identifying a $1 \times 1$ matrix with its single element. Thus the equation (6.1) becomes

$$
d P=\sigma e .
$$

Henceforth let us assume that $\mathfrak{M}$ is a manifold with an affine connection $d$. 
The operator $d$ may be applied to the displacement vector $d P$ and this leads to the following definition.

Definition 8.1. The torsion vector of $\mathfrak{M}$ is the vector $d^{2} P=d(d P)$ of the space $\mathcal{T}_{2}^{1}$.

Since each $e_{i}$ is in the space $\mathcal{T}_{0}^{1}$, we have $d e_{i} \in \mathcal{T}_{1}^{1}$. It follows that there are unique one-forms $\omega_{\imath}^{j}$ such that $d e_{i}=\sum \omega_{i}^{j} e_{j}$. This may be expressed in matrix form as

$$
d e=\Omega e, \quad \text { where } \Omega=\left\|\omega_{i}^{j}\right\| .
$$

The one-forms $\omega_{t}^{j}$ are sometimes called connection forms. If we now differentiate (8.2) we get

$$
d^{2} P=d \sigma e-\sigma d e=d \sigma e-\sigma \Omega e=\tau e, \quad \text { where } \tau=d \sigma-\sigma \Omega .
$$

Thus $\tau$ is a $1 \times n$ matrix of two-forms which we shall call the torsion forms, and we have

$$
d^{2} P=\tau e, \quad \tau=d \sigma-\sigma \Omega .
$$

Differentiating the second of these relations yields

$$
\begin{aligned}
d \tau & =-d \sigma \Omega+\sigma d \Omega=-(\tau+\sigma \Omega) \Omega+\sigma d \Omega \\
& =-\tau \Omega+\sigma\left(d \Omega-\Omega^{2}\right) .
\end{aligned}
$$

We write this as

$$
d \tau+\tau \Omega=\sigma \Theta, \quad \Theta=d \Omega-\Omega^{2} .
$$

The $n \times n$ matrix $\Theta=\left\|\theta_{i}^{\prime}\right\|$ of two forms $\theta_{i}^{j}$ is called the curvature matrix and its elements are the curvature forms of $\mathfrak{M}$. Differentiation of the second of these relations yields

$$
d \Theta=-d \Omega \Omega+\Omega d \Omega=-\left(\Theta+\Omega^{2}\right) \Omega+\Omega\left(\Theta+\Omega^{2}\right) .
$$

This gives us the known Bianchii identity

$$
d \Theta=\Omega \Theta-\Theta \Omega \text {. }
$$

It should be pointed out that this proof of (8.6) is identical with that given in Cartan [3]. It is easily verified that further differentiation of the first relation of (8.5), or of (8.6), gives no new identities.

We may remark at this point that a connection $d$ is called symmetric if its torsion vanishes. In this case, equations (8.4) and (8.5) yield the results

$$
d \sigma=\sigma \Omega, \quad \sigma \Theta=0 .
$$

Some of the results in the next section also take simpler forms when the connection is symmetric.

9. Other identities. We begin this section with the following result. 
ThEOREM 9.1 The identity

$$
d \Theta^{r}=\Omega \Theta^{r}-\Theta^{r} \Omega
$$

is valid.

This identity reduces to (8.6) for $r=1$, while if it is true for a given value of $r$, then

$$
\begin{aligned}
d\left(\Theta^{r+1}\right) & =d\left(\Theta \Theta^{r}\right)=d \Theta \Theta^{r}+\Theta d \Theta^{r} \\
& =(\Omega \Theta-\Theta \Omega) \Theta^{r}+\Theta\left(\Omega \Theta^{r}-\Theta^{r} \Omega\right) \\
& =\Omega \Theta^{r+1}-\Theta^{r+1} \Omega,
\end{aligned}
$$

hence it is true for $r+1$ and, by induction, for all $r$.

COROLlaRy 1. The following identities are valid.

$$
\begin{aligned}
& d\left(\sigma \Theta^{r}\right)=\tau \Theta^{r}+\sigma \Theta^{r} \Omega . \\
& d\left(\tau \Theta^{r}\right)=\sigma \Theta^{r+1}-\tau \Theta^{r} \Omega .
\end{aligned}
$$

These follow from (9.1), (8.4), and (8.5) by means of these computations.

$$
\begin{aligned}
& d\left(\sigma \Theta^{r}\right)=d \sigma \Theta^{r}-\sigma d \Theta^{r}=(\tau+\sigma \Omega) \Theta^{r}-\sigma\left(\Omega \Theta^{r}-\Theta^{r} \Omega\right) . \\
& d\left(\tau \Theta^{r}\right)=d \tau \Theta^{r}+\tau d \Theta^{r}=(\sigma \Theta-\tau \Omega) \Theta^{r}+\tau\left(\Omega \Theta^{r}-\Theta^{r} \Omega\right) .
\end{aligned}
$$

COROLlary 2. The result of repeated differentiation of the displacement vector $d P$ is given by

$$
d^{2 r-1} P=\sigma \Theta^{r-1} e, \quad d^{2 r} P=\tau \Theta^{r-1} e \text { for } r=1,2, \cdots .
$$

Proof. For $r=1$, the relations reduce to (8.2) and (8.4). If they are true for a given $r$, then we differentiate the second relation twice and apply (8.3) and the results of Corollary 1 to obtain

$$
\begin{aligned}
d^{2 r+1} P & =d\left(\tau \Theta^{r-1} \mathbf{e}\right)=d\left(\tau \Theta^{r-1}\right) \mathbf{e}+\tau \Theta^{r-1} d e \\
& =\left(\sigma \Theta^{r}-\tau \Theta^{r-1} \Omega\right) e+\tau \Theta^{r-1} \Omega e=\sigma \Theta^{r} \mathbf{e}, \\
d^{2 r+2} P & =d\left(\sigma^{r} \mathbf{e}\right)=d\left(\Theta^{r}\right) \mathbf{e}-\sigma^{r} d \mathbf{e} \\
& =\left(\tau \Theta^{r}+\sigma^{r} \Omega\right) e-\sigma^{r} \Omega e=\tau \Theta^{r} \mathbf{e} .
\end{aligned}
$$

Corollary 3. The identities

$$
d^{2 r-1} e=\Omega \Theta^{r-1} e, \quad d^{2 r} e=\Theta^{r} e,
$$

for $r=1,2, \cdots$, are valid.

The proof of this corollary is similar to that of the last corollary, hence we shall omit it.

We may summarize the results of this and the last section as follows. Let us regard the quantities $d P, e, \sigma, \Omega, \tau, \Theta$ as a set of basic quantities and $d$ as 
an operator which may be applied several times to any of these. Then the relations (8.2) through (9.5) express every possible result of iterated application of $d$ to any of the basic quantities as polynomials in these quantities. What is more, the application of $d$ to any of these relations yields another of them.

10. Differentiation of a vector. Let $v=\lambda^{1} e_{1}+\cdots+\lambda^{n} e_{n}$ be an arbitrary vector field, i.e., $v \in \mathcal{T}_{0}^{1}$. We set $\lambda=\left(\lambda^{1}, \cdots, \lambda^{n}\right)$ so that $\lambda$ is a $1 \times n$ matrix and $v=\lambda e$.

THEOREM 10.1. The result of repeated differentiation of $v$ is given by

$$
d^{2 r-1} \boldsymbol{V}=D \lambda \Theta^{r-1} e, \quad d^{2 r} \boldsymbol{V}=\lambda \Theta^{r} e
$$$$
\text { for } r=1,2, \cdots \text {. }
$$

Here

$$
D \lambda=\left(D \lambda^{1}, \cdots, D \lambda^{n}\right)=d \lambda+\lambda \Omega .
$$

Also, the formula

$$
d(D \lambda)=D \lambda \Omega+\lambda \Theta
$$

is valid.

Proof. If $D \lambda$ is defined by (10.2), then equation (10.3) follows by direct computation. If the first formula of (10.1) is assumed to be true for a given value of $r$-this is certainly the case for $r=1$-then differentiation yields

$$
\begin{aligned}
d^{2 r} \boldsymbol{V}= & d\left(D \lambda \Theta^{r-1} \mathbf{e}\right)=(D \lambda \Omega+\lambda \Theta) \Theta^{r-1} \mathbf{e} \\
& -D \lambda\left(\Omega \Theta^{r-1}-\Theta^{r-1} \Omega\right) e-D \lambda \Theta^{r-1} \Omega e=\lambda \Theta^{r} \mathbf{e} .
\end{aligned}
$$

We have used (9.1) in this calculation. If we differentiate again, we obtain

$$
d^{2 r+1} \boldsymbol{V}=(D \lambda-\lambda \Omega) \Theta^{r} e+\lambda\left(\Omega \Theta^{r}-\Theta^{r} \Omega\right) e+\lambda \Theta^{r} \Omega e=D \lambda \Theta^{r} e .
$$

The theorem now follows by complete induction.

The following theorem gives us an interesting application of our calculus.

Theorem 10.2. For each $r=1,2, \cdots$ and $s=1,2, \cdots$, we have

$$
\begin{aligned}
d\left(d^{2 r-1} V\right)^{s} & =s d^{2 r} V\left(d^{2 r-1} V\right)^{s-1}, \\
d\left(d^{2 r} V\right)^{2 s-1} & =d^{2 r+1} V\left(d^{2 r} V\right)^{2 s-2}, \\
d\left(d^{2 r} V\right)^{2 s} & =0 .
\end{aligned}
$$

Proof. Let us assume that we may write $d\left(d^{r} v\right)^{s}=c_{r, s} d^{r+1} v\left(d^{r} v\right)^{s-1}$, with constants $c_{r, s}$ to be determined. For $s=1$ this is possible with $c_{r, 1}=1$. From the computation

$$
\begin{aligned}
& d\left(d^{r}\right)^{*+1}=d\left[d^{r} v^{*}\left(d^{r} v^{*}\right)^{\theta}\right] \\
& =d^{r+1} v\left(d^{r} \boldsymbol{v}\right)^{\theta}+(-1)^{r} c_{r, 8} d^{r} v^{d^{r+1}} \mathbf{v}\left(d^{r} \boldsymbol{v}\right)^{s-1} \\
& =\left(1+(-1)^{r-1} c_{r, s}\right) d^{r+1} V\left(d^{r} V\right)^{s} \text {, }
\end{aligned}
$$


we see that the recursion formula

$$
c_{r, s+1}+(-1)^{r} c_{r, s}=1
$$

is satisfied and, in addition, that the form we have written is correct. One easily verifies that the solution of the recursion formula with the stated initial values is given by

$$
c_{r, 8}=\left(\frac{1-(-1)^{r}}{2}\right) s+\left(\frac{1+(-1)^{r}}{2}\right)\left(\frac{1-(-1)^{\circ}}{2}\right),
$$

and one thus obtains equations (10.4).

Thus, for example, one has the special cases

$$
d\left(d^{2} v\right)^{2}=0 \quad \text { and } \quad d\left(d^{2} v\right)^{3}=d^{3} v\left(d^{2} v\right)^{2} .
$$

Later on we shall find use for the special case $r=1$ of the first formula of (10.4) so we shall state this as a corollary.

Corollary. For $r=1,2, \cdots$, we have

$$
d(d v)^{r}=r d^{2} v(d v)^{r-1} .
$$

11. Change of frame. Let $e^{*}$ and $e$ be two moving frames defined in the same local coordinate neighborhood $\mathfrak{u}$. Then

$$
\mathrm{e}^{*}=A \mathrm{e}
$$

where $A$ is a nonsingular matrix defined on $\mathfrak{U}$. The dual bases $\sigma^{*}, \sigma$ of the given frames are related by

$$
\sigma^{*}=\sigma A^{-1}
$$

This is a consequence of $d P=\sigma e=\sigma^{*} e^{*}$. From the relation $d^{2} P=\tau e=\tau^{*} e^{*}$ we have

$$
\tau^{*}=\tau A^{-1}
$$

Next, we differentiate (11.1) to obtain

$$
\Omega^{*} e^{*}=d e^{*}=d A e+A \Omega e,
$$

and hence

$$
\Omega^{*}=A \Omega A^{-1}+d A A^{-1} .
$$

We may proceed a little differently in differentiating (11.1) to obtain the transformation law for the curvature forms. Actually we shall differentiate twice and use (9.5):

$$
d \mathrm{e}^{*}=d A \mathrm{e}+A d \mathrm{e}, \quad \Theta^{*} \mathrm{e}^{*}=d^{2} \mathrm{e}^{*}=-d A d \mathrm{e}+d A d \mathrm{e}+A d^{2} \mathrm{e}=A \Theta e .
$$

Hence we have 


$$
\Theta^{*}=A \Theta A^{-1} \text {. }
$$

Next, let $v=\lambda e$ be a vector field. Then $d v=D \lambda e=D \lambda * e^{*}$, hence

$$
D \lambda^{*}=D \lambda A^{-1} \text {. }
$$

12. Classical formulation. For the sake of completeness, we shall indicate here how the quantities we have introduced may be expressed in the language of the tensor calculus. In this section we shall use the Einstein summation convention whereby common upper and lower indices are summed.

The one-forms $\omega_{i}^{j}$ may be expressed in terms of the basis $\sigma^{k}$ of one-forms according to

$$
\omega_{i}{ }^{j}=\Gamma_{i}{ }_{k} \sigma^{k} .
$$

This defines the connection coefficients $\Gamma_{i}{ }_{i}$ relative to the given moving frame e. Similarly we may write

$$
\begin{aligned}
& \theta_{i}{ }^{j}=2^{-1} R_{i}{ }^{i}{ }_{k l} \sigma^{k} \sigma^{l} \quad \text { with } R_{i}{ }^{i}{ }_{k l}+R_{i}{ }^{i} l k=0 \text {, } \\
& \tau^{i}=2^{-1} T^{i}{ }_{j k} \sigma^{j} \sigma^{k} \quad \text { with } \quad T^{i}{ }_{j k}+T^{i}{ }_{k j}=0 \text {, } \\
& D \lambda^{i}=\lambda^{i},{ }_{j} \sigma^{j} \text {. }
\end{aligned}
$$

These equations define the Riemann tensor $R_{i}{ }^{j}{ }_{k l}$, the torsion tensor $T^{i}{ }_{j k}$, and the covariant derivative $\lambda^{i}{ }_{, j}$ of $\lambda^{i}$. That each of these quantities actually satisfies the tensor transformation law is a consequence of the equations of the last section. Each of our identities may be expressed as an identity involving the tensors defined in the last four equations.

It is now easy to show that our Definition 7.1 of an affine connection is equivalent to the usual one. We first of all specialize our formulas to the case in which the vectors $e_{1}, \cdots, e_{n}$ of the moving frame are directional differentiations with respect to coordinate functions. Thus let $x^{1}, \cdots, x^{n}$ be a local coordinate system and let

$$
e_{1}=\frac{\partial}{\partial x^{1}}, \ldots, e_{n}=\frac{\partial}{\partial x^{n}}
$$

Then by (6.1) we have

$$
d P=d x^{1} e_{1}+\cdots+d x^{n} e_{n}
$$

which is to say that $\sigma^{i}=d x^{i}$, and so $\omega_{i}{ }^{j}=\Gamma_{i}{ }^{j}{ }_{k} d x^{k}$. It follows that

$$
d e_{i}=\Gamma_{i}{ }_{k} d x^{k} e_{j}
$$

Corresponding to each frame (12.5) we thus have functions $\Gamma_{i}{ }_{i}{ }_{k}$. To find their transformation law, we let $x_{1}^{*}, \cdots, x_{n}^{*}$ be another local coordinate system and $e^{*}$ the corresponding frame. Then (11.1) is valid, where $A$ is the Jacobian matrix 


$$
A=\left\|\frac{\partial x^{j}}{\partial x^{* i}}\right\|
$$

We change the formula (11.4) to the form $\Omega^{*} A=A \Omega+d A$, and may write it as

$$
\omega^{*}{ }_{i}{ }^{i} \frac{\partial x^{k}}{\partial x^{* j}}=\frac{\partial x^{j}}{\partial x^{* i}} \omega_{j}^{k}+\frac{\partial^{2} x^{k}}{\partial x^{* i} \partial x^{* j}} d x^{* j}
$$

or

$$
\Gamma^{*}{ }_{i}^{j}{ }_{r} d x^{* r} \frac{\partial x^{k}}{\partial x^{* j}}=\frac{\partial x^{j}}{\partial x^{* i}} \Gamma_{j}{ }^{k} l \frac{\partial x^{l}}{\partial x^{* r}} d x^{* r}+\frac{\partial^{2} x^{k}}{\partial x^{* i} \partial x^{* r}} d x^{* r} .
$$

Thus we have

$$
\Gamma_{i}{ }_{r}{ }_{r} \frac{\partial x^{k}}{\partial x^{* j}}=\Gamma_{j}{ }^{k} l \frac{\partial x^{j}}{\partial x^{* i}} \frac{\partial x^{l}}{\partial x^{* r}}+\frac{\partial^{2} x^{k}}{\partial x^{* i} \partial x^{* r}} .
$$

This is precisely the formula (2.1) of Eisenhart [10].

Conversely, if we have associated with each local coordinate system $x^{1}, \cdots, x^{n}$ functions $\Gamma_{i}{ }^{j}$ which satisfy the transformation law (12.9), then we set $\omega_{i}{ }^{j}=\Gamma_{i}{ }^{j} d x^{k}, \Omega=\left\|\omega_{i}{ }^{i}\right\|$, and follow our equations backwards to formula (11.4). It then follows that if we set $d v=(d \lambda+\lambda \Omega) e$ for $v=\lambda e$, the result is independent of the particular local coordinate system with which we started and satisfies the requirements of our Definition (7.1), since the crucial formula (b) of that definition is verified by the calculation

$$
\begin{aligned}
d(f v) & =d(f \lambda e)=(d(f \lambda)+f \lambda \Omega) e \\
& =(d f \lambda+f d \lambda+f \lambda \Omega) e=d f v+f d v .
\end{aligned}
$$

We shall finally give an explicit formula for the torsion tensor $T^{i}{ }_{j k}$ in the case of a frame (12.5). From (12.6) we obtain

$$
\begin{aligned}
d^{2} P & =-d x^{i} d \boldsymbol{e}_{i}=-d x^{i} \omega_{i}{ }^{i} \boldsymbol{e}_{j}=-d x^{i} \Gamma_{i}{ }_{k} d x^{k} \boldsymbol{e}_{j} \\
& =-2^{-1}\left(\Gamma_{i}{ }^{j}{ }_{k}-\Gamma_{k}{ }^{j}{ }_{i}\right) d x^{i} d x^{k} \boldsymbol{e}_{j} .
\end{aligned}
$$

But by (8.4) and (12.3) we have

$$
d^{2} P=\tau^{j} \boldsymbol{e}_{j}=2^{-1} T^{j_{i k}} d x^{i} d x^{k} \boldsymbol{e}_{j},
$$

hence

$$
T_{i k}^{j}=\Gamma_{k}{ }_{i}{ }_{i}-\Gamma_{i}{ }^{j}{ }_{k} .
$$

Thus the tensor $T^{j}{ }_{i k}$ is essentially the skew-symmetric part (with respect to the lower indices) of the connection coefficients $\Gamma_{k}{ }^{j}$.

13. Note on tensor spaces. It is worth while mentioning that the construction of $\$ 5$ and the theorem of $\S 7$ could be formulated for tensor spaces also. 
As an example one may form the space

$$
\mathcal{f}_{q} \otimes \otimes^{p} \mathcal{T}=S_{q}^{p}
$$

This space has algebraic properties analogous to those of $\mathcal{F}_{q}^{p}$ and the affine connection $d$ induces an operator $d$ on $\mathcal{T}_{q}^{p}$ to $\mathcal{S}_{q+1}^{p}$ with properties similar to those listed in Theorem 7.1.

\section{Chapter IV. Applications to Riemannian geometry}

14. Orthonormal frames. A Riemannian space is the structure consisting of a $C^{\infty}$ manifold $\mathfrak{M}$ together with an inner product on each tangent space $\mathfrak{T}_{P}$ such that whenever $\boldsymbol{v}$ and $\boldsymbol{w}$ are vector fields, then their inner product $\boldsymbol{v} \cdot \boldsymbol{w}$ is a $C^{\infty}$ function on $\mathfrak{M}$. Locally one may always select a moving frame e such that $\boldsymbol{e}_{i} \cdot \boldsymbol{e}_{j}=\delta_{i j}$, since the Gram-Schmidt orthonormalization process involves algebraic operations only. Such a frame is called, as usual, an orthonormal frame; in the remainder of this discussion we shall deal with only this kind of frame. If $e$ and $e^{*}$ are two frames, then $e^{*}=A e$ where $A$ is an orthogonal matrix.

We may interpret the inner product as a bilinear functional on the space $\mathcal{T}_{0}^{1}$ over $\mathcal{F}_{0}$. This functional may be extended to many pairs of the spaces $\mathcal{T}_{q}^{p}$, but for our purposes only the following is needed. We define the inner product of $v \in \mathcal{T}_{0}^{1}$ and $z \in T_{q}^{1}$ by linearity and the special case $z=\eta w$, where $\eta \in \mathcal{T}_{a}^{0}, w \in \mathcal{T}_{0}^{1}$ :

$$
\boldsymbol{v} \cdot \boldsymbol{w}=\boldsymbol{z} \cdot \boldsymbol{v}=\eta(\boldsymbol{v} \cdot \boldsymbol{w}) .
$$

This is a bilinear (and commutative) operation which maps $\mathcal{T}_{0}^{1} \times \mathcal{T}_{q}^{1}$ into $\mathcal{T}_{q}^{0}$.

One of the salient features of Riemann spaces is the existence of an affine connection of a special nature.

THEOREM 14.1. There exists a unique symmetric affine connection $d$ such that

$$
d(\boldsymbol{v} \cdot \boldsymbol{w})=d \boldsymbol{v} \cdot \boldsymbol{w}+\boldsymbol{v} \cdot d \boldsymbol{w} .
$$
[11].

Proofs of this result may be found in Cartan [3], Chern [6], and Flanders

In terms of an orthonormal frame $e$ we have the relations

$$
d P=\sigma e, \quad d e=\Omega e, \quad \Omega+{ }^{t} \Omega=0, \quad d \sigma=\sigma \Omega, \quad \Theta+{ }^{t} \Theta=0 .
$$

Because of the skew-symmetry of $\Omega$ and $\Theta$, it is now advisable to write all indices as lower indices. Thus we now write $\omega_{i j}$ instead of $\omega_{i}^{j}, \theta_{i j}$ instead of $\theta_{i}^{j}$, etc.

15. The curvatura integra. In two important papers, Chern $[7,8]$ has shown how to define a series of intrinsic differential forms on the tangent bundle $\mathfrak{B}_{1}^{*}$ of all unit vectors at all points of $\mathfrak{M}$ (see $\$ 2$ above) and has given relations between them. We shall show how to express these forms in terms 
of the calculus we have developed and derive the relations systematically.

We shall assume in this section that $\mathfrak{M}$ is orientable, and shall work only with the (orthonormal) frames of a definite one of the two orientation classes of frames. Thus if $e, e^{*}$ are such frames, then $e^{*}=A e$ where $A$ is an orthogonal matrix of determinant one. It follows that $\mathbf{e}_{1}^{*} e_{2}^{*} \cdots \mathbf{e}_{n}^{*}=\mathbf{e}_{1} \cdots \mathbf{e}_{n}$, so that either of these expressions is a representation of an intrinsic $n$-vector on $\mathfrak{M}$. We shall need the following lemma.

LeMma 15.1. The following relation is true.

$$
d\left(e_{1} \cdots e_{n}\right)=0 .
$$

Proof. Since $\Omega$ is skew-symmetric, we have $d e_{1}=\omega_{12} e_{2}+\cdots+\omega_{1 n} e_{n}$. This implies that $d \mathbf{e}_{1} \mathbf{e}_{2} \cdots \mathbf{e}_{n}=0$, so the formula now follows by symmetry and equation (7.1).

From the curvature matrix $\Theta$ we can construct a new vector as follows.

Definition 15.1. The vector c of the space $\mathcal{T}_{2}^{2}$ is defined by

$$
\mathrm{c}=\sum \theta_{i j} \mathbf{e}_{i} \boldsymbol{e}_{j}={ }^{t} \mathrm{e} \Theta e .
$$

The transformation laws (11.1) and (11.5) for the case of $A$ orthogonal show us that $c$ is intrinsic.

LEMMA 15.2. $d \mathrm{c}=0$.

Proof. $d c={ }^{t} d e \Theta e+{ }^{t} e d \Theta e+{ }^{t} e \Theta d e=-{ }^{t} e \Omega \Theta e+{ }^{t} e d \Theta e+{ }^{t} e \Theta \Omega e=0$ by equation (8.6).

Now let $v$ be any unit vector in $\mathcal{T}_{0}^{1}$. We shall define two vectors that depend on $v$ alone and not on the particular frame used. We denote by $[x]$ the greatest integer in $x$.

Definition 15.2. For each $k=0,1, \cdots,[n / 2]-1$, we define $w_{k} \in \mathcal{G}_{n-1}^{n}$ and $z_{k} \in \mathcal{T}_{n}^{n}$ by

$$
\begin{aligned}
\boldsymbol{w}_{k} & =\boldsymbol{v}(d \boldsymbol{v})^{n-2 k-1} \mathbf{c}^{k}, \\
\boldsymbol{z}_{k} & =(d v)^{n-2 k-2} \mathbf{c}^{k+1} .
\end{aligned}
$$

Since $e_{1} \cdots e_{n}$ is a basis of $n$-vectors, it follows that differential forms may be defined by

$$
\begin{aligned}
\boldsymbol{w}_{k} & =\phi_{k} \mathbf{e}_{1} \cdots \boldsymbol{e}_{n}, \\
\boldsymbol{z}_{k} & =\psi_{k} \mathbf{e}_{\mathbf{1}} \cdots \boldsymbol{e}_{n} .
\end{aligned}
$$

Here $\phi_{k} \in \mathcal{F}_{n-1}$ and $\psi_{k} \in \mathcal{F}_{n}$. These are the forms $\Phi_{k}, \Psi_{k}$ of Chern [7, 8]. A basic algebraic relation is given in the following lemma.

Lemma 15.3. The following formula is valid.

$$
z_{k}=2(k+1) v d^{2} v(d v)^{n-2 k-2} c^{k} .
$$


Proof. Since $v$ is a unit vector field, we may select a moving frame $e$ in such a way that $e_{1}=v$. Since both members of (15.7) are intrinsic, we may use this particular frame in the proof. By the second equation (9.5) with $r=1$, we have $d^{2} e=\Theta e$, hence $d^{2} v=d^{2} e_{1}=\sum \theta_{1 j} e_{j}$, where the sum is taken over $j=2,3, \cdots, n$, since $\Theta$ is skew-symmetric. On the other hand, we have

$$
\begin{aligned}
\mathbf{c} & =\sum \theta_{i j} \mathbf{e}_{i} \mathbf{e}_{j}=\sum \theta_{1 j} \mathbf{e}_{1} \boldsymbol{e}_{j}+\sum \theta_{i 1} \mathbf{e}_{i} \mathbf{e}_{1}+\sum_{i, j \geqq 2} \theta_{i j} \mathbf{e}_{i} \mathbf{e}_{j} \\
& =2 \mathbf{e}_{1} \sum \theta_{1 j} \mathbf{e}_{j}+\sum_{i, j \geqq 2} \theta_{i j} \mathbf{e}_{i} \boldsymbol{e}_{j},
\end{aligned}
$$

hence $c=2 v d^{2} v+h$, where $h$ is a vector of $\mathcal{T}_{2}^{2}$ which involves only $e_{2}, \cdots, e_{n}$. Since $\boldsymbol{v}^{2}=\mathbf{0}$ and $\boldsymbol{h}$ commutes with every vector, we have

$$
\mathbf{c}^{k}=2 k \boldsymbol{v} d^{2} \boldsymbol{v} \boldsymbol{h}^{k-1}+\boldsymbol{h}^{k} \text { and } \mathbf{c}^{k+1}=2(k+1) \boldsymbol{v} d^{2} \boldsymbol{v} \boldsymbol{h}^{k}+\boldsymbol{h}^{k+1}
$$

by the binomial expansion. Next, we assert that

$$
(d v)^{n-2 k-2} h^{k+1}=0 .
$$

This is the case because $d v=d e_{1}=\sum_{j \geqq 2} \omega_{1 j} e_{j}$, and so the vector on the lefthand side is an $n$-vector in the $(n-1)$ vectors $e_{2}, \cdots, e_{n}$, and hence vanishes. Noting that $v d^{2} v$ is in $\mathcal{T}_{2}^{2}$, and so commutes with every vector, we have

$$
\begin{aligned}
\boldsymbol{z}_{k} & =(d v)^{n-2 k-2} \mathbf{c}^{k+1} \\
& =2(k+1) \boldsymbol{v} d^{2} \boldsymbol{v}(d v)^{n-2 k-2} \mathbf{h}^{k} \\
& =2(k+1) \boldsymbol{v} d^{2} v(d v)^{n-2 k-2} \mathbf{c}^{k} .
\end{aligned}
$$

The last equality is a consequence of the formula above for $c^{k}$ and the fact that $v^{2}=0$.

The following result can be read out of the last proof.

Lemma 15.4. If $\boldsymbol{v}$ is a unit vector field, then $(d v)^{n}=0$.

For if $v=e_{1}$, then $d v=\omega_{12} e_{2}+\cdots+\omega_{1 n} e_{n}$, hence $(d v)^{n}$ is an $n$-vector in $\mathbf{e}_{2}, \cdots, \boldsymbol{e}_{n}$.

It is convenient to set $\psi_{-1}=0$ and $z_{-1}=0$. We now have our main result.

Theorem 15.1. For each $k=0,1, \cdots,[n / 2]-1$, we have

$$
d \boldsymbol{w}_{k}=z_{k-1}+\frac{n-2 k-1}{2(k+1)} z_{k} .
$$

Proof. We compute $d w_{k}$ from the formula (15.3), making use of equation (10.5) and Lemma 15.2.

$$
d \boldsymbol{w}_{k}=(d v)^{n-2 k} \mathrm{c}^{k}+(n-2 k-1) v d^{2} v(d v)^{n-2 k-2} \mathrm{c}^{k} .
$$

The first term is $z_{k-1}$. This is the case by virtue of (15.4) when $k>0$, and by Lemma 15.4 when $k=0$. The second term is equal to the last term of (15.8) 
by Lemma 15.3 .

Corollary 15.1. For $k=0,1, \cdots,[n / 2]-1$, we have

$$
d \phi_{k}=\psi_{k-1}+\frac{n-2 k-1}{2(k+1)} \psi_{k} .
$$

This result now follows from (15.5), (15.6), and Lemma 15.4.

\section{BiBLIOGRAPHY}

1. N. Bourbaki, Algèbre linéaire, Paris, 1947.

2. - Algèbre multilinéaire, Paris, 1948.

3. E. Cartan, Leçons sur la géométrie des espaces de Riemann, Paris, 1928 (2d ed. 1946).

4. - Les systèmes différentiels extérieurs et leurs applications géométriques, Paris, 1945.

5. - Sur les variétés a connexion affine et la théorie de la relativité généralisée, Ann. Ecole Norm. (3) vol. 40 (1923) pp. 325-412.

6. S. S. Chern, Some new viewpoints in differential geometry in the large, Bull. Amer. Math. Soc. vol. 52 (1946) pp. 1-30.

7. - A simple intrinsic proof of the Gauss-Bonnet formula for closed Riemannian manifolds, Ann. of Math. vol. 45 (1944) pp. 747-752.

8. - On the curvatura integra in a Riemannian manifold, Ann. of Math. vol. 46 (1945) pp. $647-684$.

9. C. Chevalley, Theory of Lie groups, Princeton, 1946.

10. L. P. Eisenhart, Non-Riemannian geometry, New York, 1927.

11. H. Flanders, $A$ method of general linear frames in Riemannian geometry, I, to appear in the Pacific Journal of Mathematics.

12. N. Steenrod, The topology of fibre bundles, Princeton, 1951.

The University of California,

Berkeley, Calif. 